\title{
Playground activities and gender variation in objectively measured physical activity intensity in Australian primary school children: a repeated measures study
}

Dean A. Dudley ${ }^{1 *}$ (D) Wayne G. Cotton ${ }^{2}$, Louisa R. Peralta ${ }^{3}$ and Matthew Winslade ${ }^{4}$

\begin{abstract}
Background: Recent studies have sought to address the limited time for physical activity by focusing on increasing physical activity intensity among students during non-curricula periods and specifically school break times. We objectively measured the intensity of student physical activity (PA) during recess and lunch breaks at primary schools in the Western Sydney region of New South Wales (NSW), Australia using a 12-month repeated measures observation design study.

Methods: Systematic direct observation of recess and lunch breaks over a ten-week period in 2014 and 2015. 120 recess and lunch breaks across twenty schools (2014) with 839 periodic observations and across 15 schools with 587 periodic observations in 2015. Both observation periods were conducted over 10-weeks in Term 4 (September - December).

Results: The mean proportion of vigorous physical activity reported as a percentage (\%VPA) across both time points was $16.6 \%(S D=23.4) .36 .8 \%(S D=26.0)$ of time was spent walking and the remaining time $(46.6 \% ; S D=30$. 4) was spent in sedentary activities. There was a significant decline in \%VPA and increase in sedentary activity $(p<$ 0.01) between the two time periods of measurement. In 2014, boys spent twice as much time in \%VPA than girls during breaks in the school day and in 2015 this increased to nearly three times as much time in \%VPA. \%VPA also varied on the type of surface PA took place and the types of activities the children were allowed to undertake during breaks.

Conclusions: Recess and lunch breaks potentially offer an opportunity for children to participate in unstructured PA during the school day. Substantial variations in the \%VPA during these periods exist. Addressing playground gender participation disparities and space usability/accessibility may be a necessary first step in promoting higher PA intensities during breaks.
\end{abstract}

Keywords: Children, Health promotion, Playgrounds, New South Wales, Observational study

\footnotetext{
* Correspondence: dean.dudley@mq.edu.au

${ }^{1}$ Department of Educational Studies, Faculty of Education Studies, Macquarie

University, 1 University Ave Macquarie University NSW, Sydney 2109, Australia

Full list of author information is available at the end of the article
}

(c) The Author(s). 2018 Open Access This article is distributed under the terms of the Creative Commons Attribution 4.0 International License (http://creativecommons.org/licenses/by/4.0/), which permits unrestricted use, distribution, and reproduction in any medium, provided you give appropriate credit to the original author(s) and the source, provide a link to the Creative Commons license, and indicate if changes were made. The Creative Commons Public Domain Dedication waiver (http://creativecommons.org/publicdomain/zero/1.0/) applies to the data made available in this article, unless otherwise stated. 


\section{Background}

Evidence acquired over the last decade in Australia alone clearly demonstrates the need for increased physical activity levels and physical activity intensity in primary school-aged children [1, 2]. Current research suggests that only about half of all Australian primary school aged children are meeting the National Physical Activity and Sedentary Behaviour Recommendations [3, 4]. This is concerning considering the strong relationship between achieving the minimum of $60 \mathrm{~min}$ of physical activity each day and positive physical, social and mental health [5] and a range of academic outcomes, including cognition [6], on-task behaviour [7] and academic achievement [8].

Despite the availability of physical activity health promotion programs for primary schools, the Independent Sport Panel Report: The Future of Sport in Australia [9] and Auditor's General's report on Physical Activity in NSW Government Primary Schools [10] argue they are inadequate. Both report an insufficient allocation of school resources for children to be adequately active in terms of time and intensity (i.e. during physical education, school sport, and free play), limited teacher training in understanding the importance of physical activity and how to effectively motivate students to be active, and a lack of supportive resources for schools and primary teachers to encourage physical activity.

Recent systematic reviews have sought to examine the interventions focusing on increasing children's physical activity levels during school break times [11, 12]. These reviews report that interventions based on playground markings and equipment do increase the physical activity of schoolchildren during school break times in the short to medium term. There have been an abundance of intervention studies seeking to increase student physical activity levels during recess and lunch break times, but there have been limited studies focusing on physical activity intensity, and to the authors' knowledge no objective observational studies (free from intervention) describing the intensity of student physical activity participation over time in NSW primary schools. The primary aim of this observational study was to examine the playground activity type and gender-related variation in physical activity intensity among a large group of NSW primary school children. Additionally, we report on covariates such as surface type, temperature, school socio-economic status, and time of day to determine if these factors had any influence on physical activity intensity during recess and lunch breaks.

\section{Method}

Researchers contacted and invited 40 primary schools from the Greater Western Sydney area of NSW, Australia. This area was chosen due to the prevalence of students in this region not meeting the physical activity guidelines ${ }^{1}$. The first twenty schools to respond to the invitation were recruited into the study as this was the financial capacity of the study. All schools were Grades K-6 with a student age range of 4-12 years.

The primary outcome variable for this study was the intensity of PA levels during recess and lunch breaks as a mean percentage of time available during those periods. Simply, we were most interested in mean proportion of students engaging in vigorous physical activity (\%VPA), Walking (\%Walking), or sedentary activity (\%sedentary activity). This was measured using the System of Observing Physical Activity in Recreation and Communities (SOPARC) [13] with simultaneous observations conducted by two research assistants trained to the gold standard. Covariates included sex of student (captured by uniform observation), area type (hard or soft surface), activity type (captured using SOPARC coding of activity types), temperature (captured from the Bureau of Meteorology website), and school socio-economic status (Socio-Economic Indexes for Areas - SEIFA [14]; and Index of Community Socio-Educational Advantage - ICSEA [15]).

PA was measured using direct observation of three randomly selected recess and lunch breaks (which could range between 10 and $45 \mathrm{~min}$ ) on three separate days from each school over a 10-week period in Term 4 (September - December) of 2014 and 2015. A repeated measures design was conducted in order to reduce school variability. No other natural experiments were being conducted in the schools that remained in the study during these two observation phases. The SOPARC [13] was used to collect these data and is based on momentary time sampling procedures in which orderly and recurrent scans of individuals and contextual factors within predetermined target areas are made. iPad tablets (Apple Inc., USA) installed with the iSOPARC Application Version 1.75 (CIAFEL, Portugal: http://ciafel.fade.up.pt) were used to provide instead of the traditional paper version of the instrument. The iSOPARC is a smart device application that implements the SOPARC protocol to generate data from field observations when used by trained observers. It provided the capacity to store, process and export data in a more timely and secure manner than afforded with paper-based versions of the instrument. The iSOPARC contains a digital counter that calculates the proportion of physical activity intensity as a percentage observed time (i.e. coded as \%VPA, \%walking, or \%sedentary), the prominent activity type (i.e chasing, football, racquet games, etc....), target area mapping, project-based data management, and cloud compatible data export functions.

Boundaries for the two most commonly utilised areas for student physical activity during recess and lunch periods (as identified by the school principal or their proxy) were marked on a school map and assessed for 
suitability for iSOPARC scanning in negotiation. One undercover and one exposed area were needed for each school. Observations were only made when it was not raining, thus when the weather permitted outdoor play. Ensure consistency with observation protocol, photo documentation of all the pre-determined settings (from each school) were taken by research staff and examined in order to classify the surface type as either hard (concrete or asphalt) or soft (grass or dirt).

Four research assistants were trained as iSOPARC observers and conducted the observations. On completion of the training, the observers were only allowed to commence the observations for this study when an interrater agreement of $85 \%$ or more on all variables on pre-recorded "gold-standard" DVDs and during live field practice was reached. Thirty-two field-based inter-rater reliability checks were conducted during the 10-week observation period. During reliability checks, two observers independently coded the same students in the same lunch or recess break.

In the traditional application of the iSOPARC tool, a scan of each subject is electronically coded and identified by: sex (male or female), intensity of activity (sedentary, walking, or very active), and whether they are a child, teen, adult or senior. During a scan of each subject, the physical activity of each individual was coded as sedentary (i.e., lying down, sitting, or standing), walking, or vigorous. The activity codes used in the SOPARC instrument have been validated by heart rate monitoring of youths from kindergarten through 12 th grade $^{15}$, and by pedometry in primary school physical education classes ${ }^{15}$. Separate scans were made for females and males, and entries are also made for time of day, temperature, area accessibility, area usability and presence of supervision. Each observation was conducted twice during the recess and lunch breaks for both females and males (i.e. four observations in total for recess breaks and another four observations for lunch breaks). Additional data recorded prior to the direct observation scans included; temperature and UV level at the start and end of the observation period; whether the observation was made at recess or lunch; start and finish times of recess and lunch; and whether the area was shaded or not.

Socioeconomic status (SES) was calculated based on two Indexes (based on postcode of residence using Socio-Economic Indexes for Areas - SEIFA; and the school's Index of Community Socio-Educational Advantage - ICSEA as determined by the Australian Curriculum, Assessment and Reporting Authority).

In an effort to minimise bias, inter-rater reliability checks on $4 \%$ of the iSOPARC observations were randomised in order to prevent possible collusion. Recess and lunch break observations were randomly selected and observers and schools were given limited notice of when a reliability check was going to occur (usually less than $24 \mathrm{~h}$ ).
Ethics approval was obtained from an Australian University Human Research Ethics Committee and the NSW Department of Education. Written informed consent was provided by school principals.

Data analysis of the iSOPARC observations were analysed using Statistical Package for Social Science (SPSS) version 24. School playground physical activity was assigned as the dependent variable. Vigorous physical activity (VPA), walking and sedentary activity were the principal outcomes of interest as a mean proportion of total observations. Student sex, activity type playground surface type, temperature, SES and time of day were assigned as independent variables.

Measures of central tendency were calculated for iSOPARC data for the entire sample and then stratified by sex (boys and girls), school environment (shaded and unshaded areas) and activity type. The relationship between these covariates and activity intensity were calculated using Analysis of Covariance (ANCOVA) in SPSS version 21. The between group difference of boys and girls was calculated using an Analysis of Variance (ANOVA) in SPSS version 21.

Pearson product moment correlations were calculated on each observation for VPA based on school ICSEA, temperature (obtained via the Bureau of Meteorology website; see www.bom.gov.au), and time of day (accessed via iSOPARC). Individual schools were not included because of the variability in contextual factors in each of the different schools (i.e., population, total area, school policy around play spaces).

\section{Results}

\section{Descriptive data}

Table 1 provides an overview of the schools that consented to being in the study. The table shows that $55 \%$ of the schools in 2014 and 66\% in 2015 were located in the 5th decile (highest) of the Socio-Economic Indexes for Areas (SEIFA), with the remaining schools being in the lower deciles. However, this measure is based on postcode of the school alone and may be a misleading proxy for socio-economic status (SES) of a school population. When the Australian Curriculum, Assessment and Reporting Authority's Index of Community SocioEducational Advantage (ICSEA) [15] is used, the analysis reveals around $30 \%$ of the students enrolled in the participating schools are in the bottom quarter of the Australian population for SES, with only $20 \%$ being in the top quarter. The ICSEA may be a more indicative SES proxy for school-based studies because it calculates students' family backgrounds (parents' occupation, school education and non-school education). In addition to these student-level factors, the school's geographical location and the proportion of Indigenous students are considered when summarising educational advantage or 
Table 1 Demographic characteristics of the schools recruited in 2014 and 2015 of the study

\begin{tabular}{llll}
\hline & & 2014 & 2015 \\
\hline $\begin{array}{l}\text { Schools stratified by SEIFA } \\
\text { Index (\% of schools) based } \\
\text { on postcode of school }\end{array}$ & & & \\
& 1 & $0(0 \%)$ & $0(0 \%)$ \\
& 3 & $1(5 \%)$ & $1(7 \%)$ \\
& 4 & $3(15 \%)$ & $1(7 \%)$ \\
& 5 & $5(25 \%)$ & $3(20 \%)$ \\
$\begin{array}{l}\text { Distribution of school } \\
\text { students stratified by the ICSEA }\end{array}$ & & $11(55 \%)$ & $10(66 \%)$ \\
& Bottom Quarter & $30.6 \%$ & $28.7 \%$ \\
& Middle Quarters & $49.8 \%$ & $51.1 \%$ \\
& Top Quarter & $19.6 \%$ & $20.2 \%$ \\
\hline
\end{tabular}

disadvantage at the school level. ICSEA also provides a scale that numerically represents the relative magnitude of this influence, and is constructed taking into account both the student- and the school-level factors. Five schools were lost at the follow-up phase as they chose to participate in another study that could have potentially contaminated findings in the follow-up period.

\section{Reliability of iSOPARC in primary school settings}

Of the 60 field-based inter-rater reliability observation checks that were conducted, a high degree of reliability was found between measurements. The mean Intraclass Correlation Coefficient (ICC) for physical activity coding was .912 with a $95 \%$ confidence interval. The range of ICC was from .885 to $.932(\mathrm{~F}=11.324, p<.001)$.

\section{Main results}

During the study, we made 839 and 587 observations of student behaviour in the playground in 2014 and 2015, respectively. The fall in observations across the study was due a five schools being unavailable in 2015 as they were undertaking a separate intervention study that the researchers felt may have contaminated the results. In 2014, 425 observations occurred on hard surfaces and 414 on grass. In 2015, 284 observations occurred on hard surfaces and 302 were on grass. The mean proportion of time spent in the different intensities of PA during recess and lunch breaks can be found in Table 2 . The mean \%VPA across both time points was $16.6 \%$ $(\mathrm{SD}=23.4) .36 .8 \%(\mathrm{SD}=26.0)$ of time was spent walking and the remaining time $(46.6 \%$; $\mathrm{SD}=30.4)$ was spent in sedentary activities.

Across the observation periods, boys were significantly more active than girls during recess and lunch with boys engaging in \%VPA $22.4 \%(\mathrm{SD}=26.0 ; p<0.01)$ of their recess and lunch breaks compared with girls who only spent $10.8 \%$ of recess and lunch break in \%VPA (SD = 18.7). The percentage of time spent walking between boys and girls was comparable at $36.9 \%(\mathrm{SD}=25.4)$ and $36.8 \%$ $(\mathrm{SD}=26.6)$, respectively. Girls spent a significantly larger percentage of their recess and lunch breaks in sedentary activities $(\mathrm{p}<0.01)(52.5 \%$; $\mathrm{SD}=30.0)$, compared with boys $(40.7 \%$; $\mathrm{SD}=29.8)$.

Primary school children spent more time being vigorously active on grassed areas compared with hard surfaced areas. In fact, grassed areas were more than twice as conducive to \%VPA (23.2\%; SD =25.3), than concrete or asphalt covered surfaces (9.9\%; SD = 19.1).

Table 3 reports the differences in activity based on sex and activity type. Students (as a whole) spent the most time being vigorously active during recess and lunch when playing rugby/touch football. However, the disparity between boys and girls in this activity could only be compared in 2014, as no girls were observed playing rugby/touch football at follow-up in any of the observed schools in 2015. More boys participated compared with girls in this type of activity (57 vs 14) and were more active (44.6\%VPA vs $39.6 \% \mathrm{VPA})$ during the game. The results also indicate that boys dominate in terms of participation numbers and PA intensity in soccer, basketball and handball.

Table 4 shows that there were no significant correlations between temperature $(r=0.00 ; p=0.99)$, school ICSEA $(r=0.03 ; p=0.39)$, or time of day $(\mathrm{r}=0.00 ; \mathrm{p}=0.99)$ with student \%VPA during recess and lunch breaks (when these were treated as continuous and independent variables).

\section{Discussion}

The purpose of this study was to describe the intensity of PA primary school students participate in during recess and lunch breaks in NSW, Australia. Regarding percentage of PA intensity during recess and lunch, our main findings were that students spent the majority of their recess and lunch breaks engaged in sedentary activities. Students only spent around $17 \%$ of their recess and lunch breaks engaged in VPA and $37 \%$ of their time walking. These findings are comparable to the findings of other playground studies. The SOPLAY(System for Observing Play and Leisure Activity in Youth) [16] data from the Janssen et al.'s (2013) study conducted in the Netherlands [17] reported that primary school children in the control group were engaged in moderate to vigorous physical activity for $38.7 \%$ of recess and lunch breaks. In Australia, Willenberg and colleagues ${ }^{18}$ also used SOPLAY, with the percentage of children participating in sedentary, moderate and vigorous activity being 44\%, 30\% and $27 \%$, respectively, during recess and lunch breaks. 


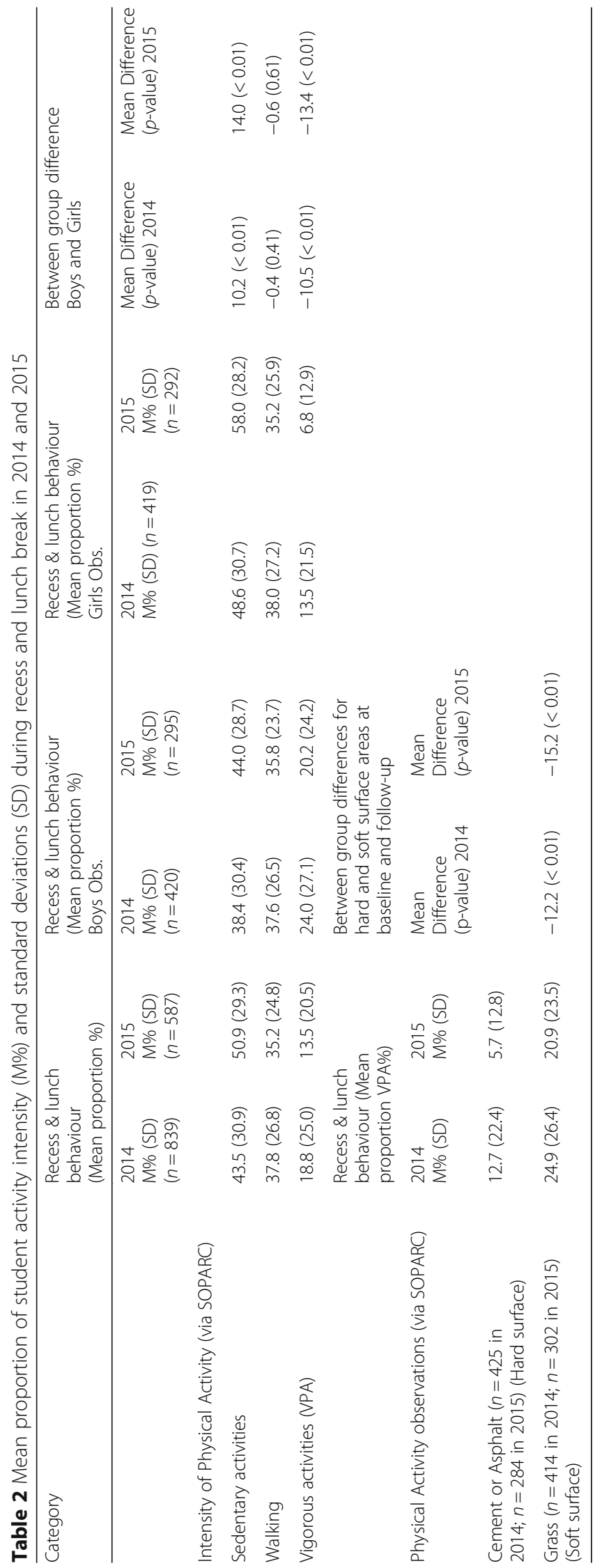




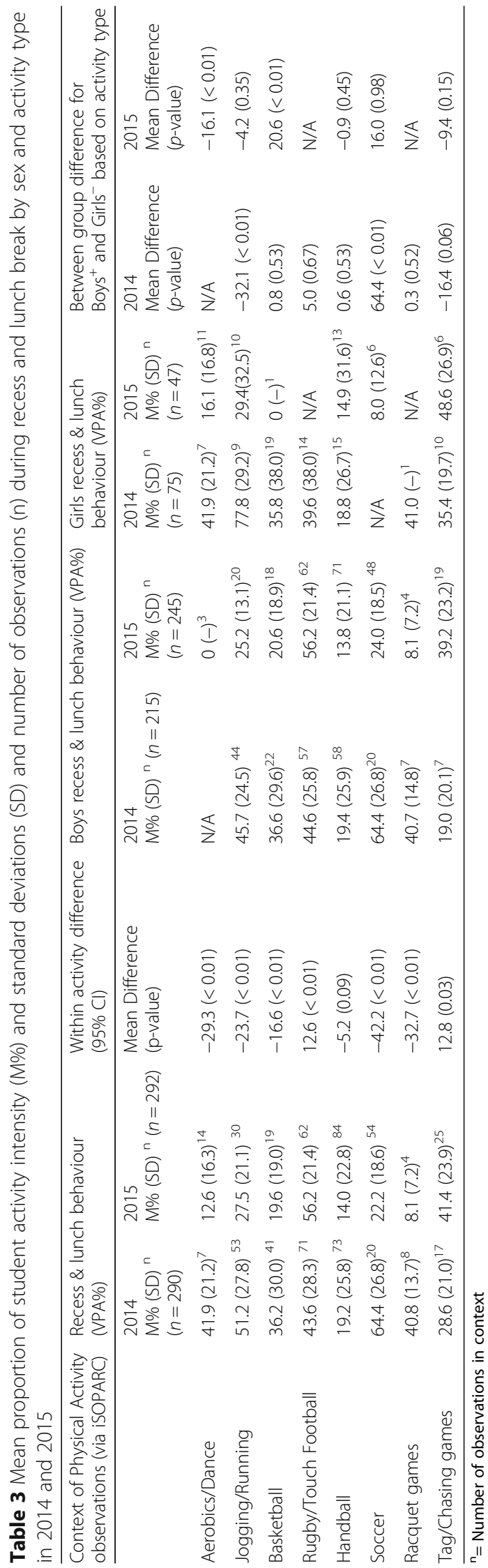


Table 4 Correlations of varying contexts with vigorous physical activity during recess and lunch breaks

\begin{tabular}{llc}
\hline & \multicolumn{2}{l}{ Observations $(n=1426)$} \\
\hline $\begin{array}{l}\text { Environmental factors (PC) } \\
\text { Temperature }\end{array}$ & 0.00 & $p$ value \\
School factors (PC) & & 0.99 \\
School ICSEA & 0.03 & 0.39 \\
Time of day (PC) & & \\
1000 h -1400 h (EDST) & 0.00 & 0.99 \\
\hline
\end{tabular}

PC Pearson correlation; ICSEA Index of Community Socio-Educational Advantage; ESDT Eastern Daylight Savings Time (Australia)

These direct observation results are comparable with the behaviour patterns observed in this study.

When examining the data by sex, girls' spent half the amount of time engaged in VPA (11\%) when compared with boys (22\%). There was also a gender difference in the percentage of recess and lunch breaks spent in sedentary activities (girls 53\%; boys $41 \%$ ). Whilst conducted in another Australian capital city, Willenberg et al. [18] reported a comparable difference in gender-based \%VPA (Boys $=32 \%$; Girls $=22 \%$ ) and sedentary activities (Boys 39\%; Girls 49\%) during recess and lunch breaks.

The findings of a discrepancy in the intensity of PA between boys and girls at recess and lunch are clear and worthy of further investigation. Blatchford et el [19] argues that the 'social' dynamic of the playground may influence a child's capacity to engage in more VPA. According to Blatchford and colleagues [19], the school playground is an important social setting, especially for boys. In this study, 582 observations of VPA during recess and lunch breaks, exclusively came from team sport participation (i.e. basketball, rugby/touch, or soccer) $(n=267)$ in which boys participated more frequently and in greater intensity. However, apart from the participation rates, the differences in intensity were only significant in basketball $(p<0.01)$. Future interventions may benefit from investigating the participation in team sports during recess and lunch breaks and providing spaces for these activities to be conducted.

This study also shows that a small number of girls were, at some point, involved in ball games such as rugby/touch football and basketball. However, girls were more likely to engage in tag/chasing games or aerobics/ dance activities than boys. These are games where students are less likely to bump into each other or have boys dominate the activity or intensity. So although there were certainly some girls interested in football and basketball, other girls enjoyed playing other kinds of games with less physical collision (and less PA intensity).
Given the opportunity, girls may participate in ball games, but might well avoid others that involve physical collisions or boy domination (i.e. football) [19].

Another explanation for the lack of \%VPA during recess and lunch by girls may be associated with the requirement for all children at these schools to wear school uniforms. School uniforms have been explained by a small number of quantitative and a larger number of qualitative studies which have shown that having to wear tight, ill-fitting, gender stereotyped or uncomfortable uniforms were major barriers to girls participating in school-based physical activity [20-22] and also may affect physical self-esteem[22]. As such, Dudley and colleagues ${ }^{20}$ recommended that schools policy makers reconsider the daily uniform students are required to wear if they are going to engage with physical education and participate in regular school-based PA.

It has also been proposed in the literature that differences in physical activity participation between boys and girls are more apparent in unstructured setting environments (such as recess and lunch breaks at school) [23]. Our study supports for this finding and therefore suggests that primary school recess and lunch breaks are a particularly important setting for investigating why these discrepancies exist, which is highlighted as we know differences in fundamental movement skills performance and physical activity participation between the sexes widen as they move into adolescence[24]. During primary school skill differences are generally determined by contextual rather than physiological factors[25], hence cultural and environmental factors of a primary school playground may be inhibiting PA intensity of girls compared with boys [26].

Grassed areas were more conducive to \%VPA with twice as much \%VPA occurring in those areas when compared with hard surfaced areas during recess and lunch breaks. These findings are new and in stark contrast to other Australian playground studies. Willenberg et al. ${ }^{18}$ reported no statistical difference between \%VPA on hard and soft surfaces (29\%; $27 \%$ respectively). These findings indicate that there are perhaps differences in school policy in NSW and Victoria around play in these areas. An explanation for this behaviour pattern could be attributed to play experiences in NSW primary schools being limited for many children due to excessive fear of risk, teacher encouragement or 'surplus safety'[27] policies compared with Victorian primary schools. There is an increasing body of evidence to suggest safety-related policies regarding school playground behaviour and the role teachers take in enforcing or encouraging behaviour will restrict or enhance the quantity and intensity of PA children undertake during recess and lunch breaks [27]. 
Contrary to previous research conducted in Australia $[12,18]$, we found no significant or meaningful relationships between temperature, school socio-economic status, or time of day with the \%VPA students engage in during recess and lunch breaks. In terms of temperature, this may be that there was in fact little variation (approximately 10 degrees centigrade) at any given data collection point where previous studies conducted more seasonally based analysis where temperature variation would be greater. To our knowledge, this is the first study that has used the ICSEA scale as a proxy of school socio economic status in an analysis of recess and lunch break physical activity free of intervention. It may be that the lack of sensitivity of ICSEA and iSOPARC to detect specific individual physical activity patterns and their socio-economic status that make this analysis incongruent. Time of day analysis based on this study appears to also be irrelevant.

There are some strengths and limitations associated with the use of the iSOPARC method when measuring intensity of PA in children in schools settings. The first limitation is that iSOPARC requires the observer to conduct scans of the observation area and code the intensity type in quick succession. The dynamic nature of children in a school playground wearing similar uniforms means that the possibility of double coding an individual may occur. This limitation is exacerbated with larger observation areas and more students. A second limitation is the ability of the iSOPARC application to replicate multiple site settings across tablet devices. These currently need to be done individually and thus risk potential data entry error. However, these limitations were offset by the fact that iSOPARC assisted researchers by providing a reliable, efficient and user-friendly means of data collection. Other limitations of the iSOPARC data were its inability to provide accurate estimates of moderate to vigorous PA which make comparisons to some national guidelines difficult. For this reason, we focussed specially on VPA which in turn may be perceived by some as limitation to generalizability. Finally, the inability to control the periods of time spent in recess and lunch breaks made comparable analysis of minutes spent in PA impossible.

\section{Conclusion}

This study shows that recess and lunch breaks are one of the few spaces in which some students can accrue unstructured PA during the school day. The \%VPA across both time points was only $16.6 \%(\mathrm{SD}=23.4)$ with the majority of the remaining time $(46.6 \%$; $\mathrm{SD}=30.4)$ being spent in sedentary activities. Boys are spending up to three times as much time in \%VPA compared with girls suggesting that school playgrounds either facilitate or inhibit participation based on sex. \%VPA also varied significantly based on the type of surface $(p<0.01)$ and the types of activities the children $(p<0.01)$ were allowed to undertake during recess and lunch.

\section{Abbreviations}

SOPARC: System of Observing Physical Activity in Recreation and

Communities (Application version); ICSEA: Index of Community SocioEducational Advantage; NSW: New South Wales; PA: Physical activity;

SEIFA: Socio-Economic Indexes for Areas; SOPARC: System of Observing Physical Activity in Recreation and Communities; SOPLAY: System for Observing Play and Leisure Activity in Youth; SPSS: Statistical Package for Social Science; VPA: Vigorous physical activity

\section{Acknowledgements \\ The authors are grateful for the support and cooperation of the participating schools, students, and teachers from the NSW Department of Education. We also wish to acknowledge the funding of this project from the NSW Cancer Institute and Cancer Council NSW.}

\section{Competing of interest}

The authors have no conflicts of interest to declare.

\section{Funding}

The funds for this project were made available through the NSW Cancer Institute's Evidence to Practice Grant and Cancer Council NSW (MQU IRIS No: 9201401102).

\section{Availability of data and materials}

As per ethics committee instructions, all data and materials for this study must remain in the secure possession of the researchers for a period of seven years. For this reason, the data cannot be shared.

\section{Authors' contributions}

DD, MW, LP and WC obtained funding for the research. All authors contributed to reviewing, editing and approving the final version of the paper. DD is the guarantor and accepts responsibility for the conduct of the study. All authors have read and approved the final manuscript.

\section{Ethics approval and consent to participate}

Ethics approval was obtained from the Charles Sturt University Human Ethics Committee (HREC 2014/062) and the New South Wales Department of Education (SERAP: 2014148). Written consent was also obtained from all school principals for the observations to occur in their respective schools and parents/guardians of students at schools were informed of the school's consent and the nature of the study via school newsletters (both paper and electronic). This paper represents a secondary analysis of data collected from a study that is registered with the Australian and New Zealand Clinical Trials Registry (ACTRN12614000926639).

Consent for publication

Not applicable.

\section{Publisher's Note}

Springer Nature remains neutral with regard to jurisdictional claims in published maps and institutional affiliations.

\section{Author details}

${ }^{1}$ Department of Educational Studies, Faculty of Education Studies, Macquarie University, 1 University Ave Macquarie University NSW, Sydney 2109, Australia. ${ }^{2}$ Sydney School of Education and Social Work, University of Sydney. University of Sydney NSW 2006, Sydney, Australia. ${ }^{3}$ Sydney School of Education and Social Work, University of Sydney. University of Sydney NSW 2006, Sydney, Australia. ${ }^{4}$ School of Teacher Education, Charles Sturt

University. Panorama Ave Bathurst, Bathurst, NSW, Australia.

Received: 31 January 2018 Accepted: 30 August 2018

Published online: 10 September 2018

\section{References}

1. Hardy L, King L, Espinel P, et al. NSW Schools Physical Activity and Nutrition Survey (SPANS) 2010: Full Report. 2013 May 20.

2. Drummond M, Drummond C, Dollman J, et al. Physical activity from early childhood to adolescence: a literature review of issues and interventions in disadvantaged populations. J Student Wellbeing. 2011 Jan 31;4(2):17-31. 
3. Okely AD, Salmon J, Vella SA, et al A Systematic Review to update the Australian Physical Activity Guidelines for Children and Young People. Canberra: Report prepared for the Australian Government Department of Health; 2012.

4. Okely AD, Salmon J, Vella SA, et al. A systematic review to inform the Australian sedentary behaviour guidelines for children and young people. Canberra: Report prepared for the Australian Government Department of Health; 2012.

5. Strong WB, Malina RM, Blimkie CJ, Daniels SR, Dishman RK, Gutin B, et al. Evidence based physical activity for school-age youth. The Journal of paediatrics. 2005;146(6):732-7.

6. Chaddock $L$, Pontifex MB, Hillman $\mathrm{CH}$, Kramer AF. A review of the relation of aerobic fitness and physical activity to brain structure and function in children. J Int Neuropsychol Soc. 2011;17:975-85.

7. Grieco LA, Jowers EM, Errisuriz VL, Bartholomew JB. Physically active vs. sedentary academic lessons: a dose response study for elementary student time on task. Prev Med. 2016:89:98-103.

8. Donnelly JE, Greene JL, Gibson CA, Smith BK, Washburn RA, Sullivan DK, et al. Physical activity across the curriculum (PAAC): a randomized controlled trial to promote physical activity and diminish overweight and obesity in elementary school children. Prev Med. 2009;49:336-41.

9. Australian Government Independent Sport Panel (2009) The Future of Sport in Australia (Crawford Report). Available at: http://apo.org.au/node/19766.

10. New South Wales Auditor-General's Report Performance Audit: Physical Activity in Government Primary Schools Department of Education and Communities. 2012, Sydney, NSW: Audit Office of NSW, Editor.

11. Escalante Y, García-Hermoso A, Backx K, Saavedra JM. Playground designs to increase physical activity levels during school recess: a systematic review. Health Educ Behav. 2014 Apr;41(2):138-44.

12. Parrish $A M$, Okely AD, Stanley RM, Ridgers ND. The effect of school recess interventions on physical activity. Sports Med. 2013 Apr 1;43(4): 287-99.

13. McKenzie $T L$, Cohen DA, Sehgal A, et al. System for observing play and recreation in communities (SOPARC): reliability and feasibility measures. J Phys Act Health. 2006 Feb;3(s1):S208-22.

14. Trewin D. Socio-economic indexes for areas (SEIFA). Canberra, Australia: Australian Bureau of Statistics; 2001

15. Louden B. Index of community socio-educational advantage [ICSEA]. In: In Socioeconomic Status and Higher Education Students Symposium, University of Technology of Sydney. Sydney; 2010 Apr. (Vol. 12).

16. McKenzie TL. SOPLAY system for observing play and leisure activity in youth, description and procedures manual. San Diego, CA: Universidad Estatal de San Diego; 2006.

17. Janssen M, Twisk JW, Toussaint HM, et al. Effectiveness of the PLAYgrounds programme on PA levels during recess in 6-year-old to 12-year-old children. Brit J Sports Med. 2013; bjsports-2012

18. Willenberg LJ, Ashbolt R, Holland D, et al. Increasing school playground physical activity: a mixed methods study combining environmental measures and children's perspectives. J Sci Med Sport. 2010 Mar 31; 13(2):210-6

19. Blatchford P, Baines E, Pellegrini A. The social context of school playground games: sex and ethnic differences, and changes over time after entry to junior school. Brit J Dev Psych. 2003 Nov 1;21(4):481-505.

20. Dudley DA, Pearson P, Okely AD, et al. Recommendations for policy and practice of physical education in culturally and linguistically diverse Australian secondary schools based on a two-year prospective cohort study. Sch Psy Int. 2015 Apr;36(2):172-88.

21. Campagna P, Ness GW, Murphy RJ, et al. Physical activity levels in children and youth in the province of Nova Scotia. Sport and Recreation Commission, Government of Nova Scotia; 2002 Dec.

22. Coakley J, White A. Making decisions: gender and sport participation among British adolescents. Socio Sport J. 1992 Mar:9(1):20-35.

23. Weiss MR. Motivating kids in physical activity. Pres Council Phys Fit Sports Res Digest 2000 Sep.

24. Eaton WO, Enns LR. Sex differences in human motor activity level. Psych Bull. 1986;100(1):19.

25. McKenzie TL, Sallis JF, Elder JP, et al. Physical activity levels and prompts in young children at recess: a two-year study of a bi-ethnic sample. Res Q Exerc Sport. 1997;68(3):195-202.
26. Thomas, J. R., Thomas, K. T., \& Gallagher, J. D. (1993). Developmental considerations in skill acquisition. In R.N. Singer, M. Murphey, \& L. K.Tennant (Eds.), Handbook of research on sport psychology (pp. 73-105). New York: Macmillan.

27. Wyver S, Tranter P, Naughton G, et al. Ten ways to restrict children's freedom to play: the problem of surplus safety. Cont Iss Early Child. 2010 Sep;11(3):263-77.
Ready to submit your research? Choose BMC and benefit from:

- fast, convenient online submission

- thorough peer review by experienced researchers in your field

- rapid publication on acceptance

- support for research data, including large and complex data types

- gold Open Access which fosters wider collaboration and increased citations

- maximum visibility for your research: over $100 \mathrm{M}$ website views per year

At BMC, research is always in progress.

Learn more biomedcentral.com/submissions 